\title{
The Nonlocal Fractal Integral Reverse Minkowski's and Other Related Inequalities on Fractal Sets
}

\author{
Gauhar Rahman $\mathbb{D}^{\mathbb{D}},{ }^{1}$ Kottakkaran Sooppy Nisar $\mathbb{D}^{2},{ }^{2}$ and Alireza Khalili Golamankaneh $\mathbb{i D}^{3}$ \\ ${ }^{1}$ Department of Mathematics and Statistics, Hazara University, Mansehra, Pakistan \\ ${ }^{2}$ Department of Mathematics, College of Arts and Science, Prince Sattam bin Abdulaziz University, \\ Wadi Aldawaser 11991, Saudi Arabia \\ ${ }^{3}$ Young Researchers and Elite Club, Islamic Azad University, Urmia Branch, Urmia, Iran \\ Correspondence should be addressed to Kottakkaran Sooppy Nisar; ksnisar1@gmail.com
}

Received 17 August 2020; Revised 23 January 2021; Accepted 28 January 2021; Published 16 February 2021

Academic Editor: Giovanni Falsone

Copyright (c) 2021 Gauhar Rahman et al. This is an open access article distributed under the Creative Commons Attribution License, which permits unrestricted use, distribution, and reproduction in any medium, provided the original work is properly cited.

In this paper, we study the generalized Riemann-Liouville fractional integral for the functions with fractal support. The aim of this article is to investigate reverse Minkowski's inequalities and certain other related inequalities by employing the generalized Riemann-Liouville fractional integral for the functions with fractal support.

\section{Introduction}

Fractional calculus involves integrals and derivatives of arbitrary order. The applications of fractional calculus have been found in the field of several sciences and engineering $[1-8]$. In $[1,2]$, the nonlocal fractional integrals and derivatives are utilized to model the processes with memory effect. In [9-11], the researchers used the nonlocal derivatives to model more appropriately the dynamics of the nonconservative systems in formulation of Hamilton and Lagrange.

Fractal analysis has been studied by many researchers by using measure theory, harmonic analysis, stochastic process, fractional spaces, and other techniques [12-35].

Recently, Parvate and Gangal [36-41] proposed the $\mathscr{F}^{\alpha}$-calculus on the fractal subset of real line and fractal curves. The researchers have applied transport materials on disordered systems such as fractal sets and fractal curves [42-44]. In [45], the researchers established Schrödinger's equation on a fractal curve.

Such new developments in fractional calculus encourage future research to investigate new innovative ideas to unify the fractional operators and establish inequalities involving new fractional operators. The fractional integral inequalities
(FII in short) and their applications play an important role in the field of applied mathematics. A wide number of integral inequalities and their extensions were built in the sense of classical fractional integral and fractional derivative operators (see, e.g., [46-50]).

The inequalities, especially the Hölder, the reverse Minkowski, the arithmetic, and geometric inequalities, have played a key role in the field of both pure as well as applied mathematics. These and several other essential inequalities are now in common use and, therefore, it is not surprising that several studies associated to these areas have been made in order to accomplish a diversity of desired goals. In the past few decades, the theory of inequalities has established rapidly and unexpected results were investigated, along with simpler new proofs for existing results, and, accordingly, new direction for research has been opened up. Recently, the theory of inequalities has gained more considerable interest from many mathematicians, and a large number of new inequalities have been estimated in the literature. It is recognized that, in general, some specific inequalities provide a useful and important device in the development of different branches of mathematics. In [51], Dahmani has investigated the reverse Minkowski fractional integral inequalities. Sousa and Capelas de Oliveira [52] have 
investigated the reverse Minkowski inequalities and certain other related inequalities for Katugampola fractional Integral operators. In $[53,54]$, the authors have studied the reverse Minkowski inequalities by considering Hadamard fractional integral operators. In this present article, we study the said inequalities by considering the generalized Riemann-Liouville fractional integral for the functions with fractal support.

The structure of the paper is follows.

In Section 2, we have given some known results and basic definitions. In Section 3, the nonlocal reverse Minkowski inequalities are presented for nonlocal fractal integrals on fractal subset of real line. In Section 4, some other related inequalities for nonlocal fractal integrals on fractal subset of real line are presented.

\section{Preliminaries}

Some well-known basic definitions and results associated with classical fractional integrals and generalized fractional integrals are presented in this section. The reverse Minkowski's integral inequalities can be found in the work of $[27,55]$. The reverse Minkowski's inequalities are the motivation of the work performed so far, involving the classical Riemann integrals which are presented by the following theorems.

Theorem 1 (see [55]). Let the two functions $\mathcal{U}_{1}$ and $\mathcal{U}_{2}$ be positive on $[0, \infty)$ and $\delta \geq 1$. If $0<k \leq\left(\mathcal{U}_{1}(\zeta) / h_{1}(\zeta)\right) \leq K$, $\zeta \in\left[x_{1}, x_{2}\right]$, then the following inequality holds:

$$
\begin{aligned}
& \left(\int_{x_{1}}^{x_{2}} \mathcal{U}_{1}^{\delta}(\zeta) \mathrm{d} \zeta\right)^{(1 / \delta)}+\left(\int_{x_{1}}^{x_{2}} \mathcal{U}_{2}^{\delta}(\zeta) \mathrm{d} \zeta\right)^{(1 / \delta)} \\
& \quad \leq \frac{1+K(k+2)}{(k+1)(K+1)}\left(\int_{x_{1}}^{x_{2}}\left(\mathcal{U}_{1}+\mathcal{U}_{2}\right)^{\delta}(\zeta) \mathrm{d} \zeta\right)^{(1 / \delta)}
\end{aligned}
$$

Theorem 2 (see [55]). Let the two functions $\mathcal{U}_{1}$ and $\mathscr{U}_{2}$ be positive on $[0, \infty)$ and $\delta \geq 1$. If $0<k \leq\left(\mathcal{U}_{1}(\zeta) / h_{1}(\zeta)\right) \leq K$, $\zeta \in\left[x_{1}, x_{2}\right]$, then the following inequality holds:

$$
\begin{gathered}
\left(\int_{x_{1}}^{x_{2}} \mathcal{u}_{1}^{\delta}(\zeta) \mathrm{d} \zeta\right)^{(2 / \delta)}+\left(\int_{x_{1}}^{x_{2}} \mathcal{u}_{2}^{\delta}(\zeta) \mathrm{d} \zeta\right)^{(2 / \delta)} \\
\geq\left(\frac{(K+1)(k+1)}{K}-2\right)\left(\int_{x_{1}}^{x_{2}} \mathcal{u}_{1}^{\delta}(\zeta) \mathrm{d} \zeta\right)^{(1 / \delta)} \\
\cdot\left(\int_{x_{1}}^{x_{2}} \mathcal{U}_{2}^{\delta}(\zeta) \mathrm{d} \zeta\right)^{(1 / \delta)} .
\end{gathered}
$$

Definition 1 (see $[5,6])$. The well-known classical fractional integrals of order $\lambda>0$ are, respectively, defined by

$$
\left({ }_{x_{1}} \mathfrak{T}^{\lambda} \mathcal{U}_{1}\right)(\zeta)=\frac{1}{\Gamma(\lambda)} \int_{x_{1}}^{\zeta}(\zeta-\rho)^{\lambda-1} \mathscr{U}_{1}(\rho) d \rho, \quad x_{1}<\zeta,
$$

and

$$
\left(\mathfrak{T}_{x_{2}}^{\lambda} \mathcal{U}_{1}\right)(\zeta)=\frac{1}{\Gamma(\lambda)} \int_{\zeta}^{x_{2}}(\rho-\zeta)^{\lambda-1} g(\rho) d \rho, \quad \zeta<x_{2},
$$

where $\lambda \in \mathbb{C}$ with $\mathfrak{R}(\lambda)>0$.

Dahmani [51] has investigated the following inequalities by using classical fractional integral.

Theorem 3 (see [51]). Let the two functions $\mathcal{U}_{1}$ and $\mathcal{U}_{2}$ be positive on $[0, \infty)$ such that, for all $\zeta>0, \mathfrak{I}^{\tau} \mathcal{U}_{1}^{\delta}(\zeta)<\infty$, $\mathfrak{I}^{\tau} \mathcal{U}_{2}^{\delta}(\zeta)<\infty$. If $0<k \leq\left(\mathcal{U}_{1}\left(\rho_{1}\right) / \mathcal{U}_{2}\left(\rho_{1}\right)\right) \leq K, \rho_{1} \in\left[x_{1}, \zeta\right]$, then the following inequality holds:

$$
\begin{aligned}
& \left(\mathfrak{T}^{\tau} \mathscr{U}_{1}^{\delta}(\zeta)\right)^{(1 / \delta)}+\left(\mathfrak{T}^{\tau} \mathcal{U}_{2}^{\delta}(\zeta)\right)^{(1 / \delta)} \\
& \quad \leq \frac{1+K(k+2)}{(k+1)(K+1)}\left(\mathfrak{T}^{\tau}\left(\mathcal{U}_{1}+\mathscr{U}_{2}\right)^{\delta}(\zeta)\right)^{(1 / \delta)},
\end{aligned}
$$

$\tau \in \mathbb{C}, \mathfrak{R}(\tau)>0, \delta \geq 1$.

Theorem 4 (see [51]). Let the two functions $\mathcal{U}_{1}, \mathscr{U}_{2}$ be positive on $[0, \infty)$ such that, for all $\zeta>0, \mathfrak{I}^{\tau} \mathcal{U}_{1}^{\delta}(\zeta)<\infty$, $\mathfrak{I}^{\tau} \mathcal{U}_{2}^{\delta}(\zeta)<\infty$. If $0<k \leq\left(\mathcal{U}_{1}\left(\rho_{1}\right) / \mathcal{U}_{2}\left(\rho_{1}\right)\right) \leq K, \rho_{1} \in\left[x_{1}, \zeta\right]$, then the following inequality holds:

$$
\begin{aligned}
& \left(\mathfrak{I}^{\tau} \mathcal{U}_{1}^{\delta}(\zeta)\right)^{(2 / \delta)}+\left(\mathfrak{I}^{\tau} \mathcal{U}_{2}^{\delta}(\zeta)\right)^{(2 / \delta)} \\
& \quad \geq\left(\frac{(K+1)(k+1)}{K}-2\right)\left(\mathfrak{I}^{\tau} \mathcal{U}_{1}^{\delta}(\zeta)\right)^{(1 / \delta)}\left(\mathfrak{I}^{\tau} \mathcal{U}_{2}^{\delta}(\zeta)\right)^{(1 / \delta)},
\end{aligned}
$$

$\tau \in \mathbb{C}, \mathfrak{R}(\tau)>0, \delta \geq 1$

In [1], it is shown that the geometry of fractal is the geometry of real world. In [36, 37, 39], Parvate and Gangal proposed the calculus on fractals which is related to Riemann integrals.

Definition 2 (see [36, 37, 39]). For the thin Cantor set, the following integral staircase function is defined by

$$
S_{\mathscr{F}}^{\alpha}(\rho)= \begin{cases}\gamma^{\alpha}\left(\mathscr{F}, x_{1}, x_{2}\right), & \text { if } \rho \geq x_{0}, \\ -\gamma^{\alpha}\left(\mathscr{F}, x_{1}, x_{2}\right), & \text { otherwise, }\end{cases}
$$

where $\alpha$ is the $\gamma$-dimension of thin Cantor set.

Definition 3 (see $[36,37,39])$. The $\mathscr{F}^{\alpha}$-derivative is defined by

$$
\mathscr{D}_{\mathscr{F}}^{\alpha} \mathcal{U}(x)= \begin{cases}\mathscr{F}-\lim _{t \rightarrow x} \frac{\mathcal{U}(t)-\mathcal{U}(x)}{S_{\mathscr{F}}^{\alpha}(t)-S_{\mathscr{F}}^{\alpha}(x)}, & \text { if } x \in \mathscr{F}, \\ 0, & \text { otherwise }\end{cases}
$$

when the limit exists. 
Definition 4. The Gamma function with the fractal support is defined by

$$
\Gamma_{\mathscr{F}}^{\alpha}(\tau)=\int_{0}^{\infty} e^{-S_{\mathscr{F}}^{\alpha}(x)} S_{\mathscr{F}}^{\alpha}(x)^{S_{\mathscr{F}}^{\alpha}(\tau)-1} d_{\mathscr{F}}^{\alpha} x,
$$

where $e^{-S_{\mathscr{F}}^{\alpha}(x)}=\mathscr{F}-\lim _{m \longrightarrow \infty}\left(1-\left(S_{\mathscr{F}}^{\alpha}(t) / m\right)\right)^{m}$.
Here, we review the following nonlocal fractal integral operators for the functions with fractal support $[56,57]$.

Definition 5. If $\mathcal{U}_{1}(x) \in C_{\mathscr{F}}^{\alpha}\left[x_{1}, x_{2}\right]$ ( $\alpha$-order differentiable function on $\left.\left[x_{1}, x_{2}\right]\right)$ and $\tau>0$, then the left- and right-sided Riemann-Liouville fractal integral operators of order $\tau$ are, respectively, defined by $[56,57]$

$$
\left({ }_{x_{1}} \mathfrak{T}^{\tau} g\right)(\zeta)=\frac{1}{\Gamma_{\mathscr{F}}^{\alpha}(\tau)} \int_{x_{1}}^{\zeta}\left(S_{\mathscr{F}}^{\alpha}(\zeta)-S_{\mathscr{F}}^{\alpha}(\rho)\right)^{\tau-\alpha} \mathcal{U}_{1}(\rho) d_{\mathscr{F}}^{\alpha} \rho, S_{\mathscr{F}}^{\alpha}\left(x_{1}\right)<S_{\mathscr{F}}^{\alpha}(\zeta)
$$

and

$$
\left(\mathfrak{T}_{x_{2}}^{\tau} g\right)(\zeta)=\frac{1}{\Gamma_{\mathscr{F}}^{\alpha}(\tau)} \int_{\zeta}^{x_{2}}\left(S_{\mathscr{F}}^{\alpha}(\rho)-S_{\mathscr{F}}^{\alpha}(\zeta)\right)^{\tau-\alpha} \mathscr{U}_{1}(\rho) d_{\mathscr{F}}^{\alpha} \rho, S_{\mathscr{F}}^{\alpha}(\zeta)<S_{\mathscr{F}}^{\alpha}\left(x_{2}\right)
$$

where $\Gamma_{\mathscr{F}}^{\alpha}(\tau)$ is defined in (7), $S_{\mathscr{F}}^{\alpha}$ is the staircase function, and $\mathscr{F}$ is the fractal set with $\alpha$-dimension (see, e.g., $[56,57]$ ).

Remark 1. If we consider $\alpha=1$ in (10) and (11), then we get (3) and (4), respectively.

One can easily prove the following lemma $[56,57]$.

\section{Lemma 1.}

$$
\begin{aligned}
& \mathfrak{T}_{x_{1}}^{\tau}\left(S_{\mathscr{F}}^{\alpha}(\zeta)-S_{\mathscr{F}}^{\alpha}\left(x_{1}\right)\right)^{\lambda} \\
& \quad=\frac{\Gamma_{\mathscr{F}}^{\alpha}(\lambda+1)}{\Gamma_{\mathscr{F}}^{\alpha}(\lambda+\tau+1)}\left(S_{\mathscr{F}}^{\alpha}(\zeta)-S_{\mathscr{F}}^{\alpha}\left(x_{1}\right)\right)^{\lambda+\tau}, \quad \lambda>-1 .
\end{aligned}
$$

\section{The Nonlocal Reverse Minkowski Inequalities on Fractal Sets}

In this section, we present the nonlocal fractal reverse Minkowski integral inequalities in the fractal support by using the generalized nonlocal fractal integral operator. The nonlocal reverse Minkowski fractal integral inequalities in fractal support are presented in the following theorems.

Theorem 5. Let $\tau \in \mathbb{C}, \mathfrak{R}(\tau)>0, \delta \geq 1$, and $\mathcal{U}_{1}$ and $\mathcal{U}_{2} \in C_{\mathscr{F}}^{\alpha}\left[x_{1}, x_{2}\right], \quad$ ( $\alpha$-order differentiable functions on $\left.\left[x_{1}, x_{2}\right]\right)$ be two positive functions on $[0, \infty)$ such that, for all theta $>0, \quad x_{1} \mathfrak{T}^{\tau} g_{1}^{\delta}(\zeta)<\infty$ and ${ }_{x} \mathfrak{T}^{\tau} \mathcal{U}_{2}^{\delta}(\zeta)<\infty$. If $0<k \leq\left(\mathscr{U}_{1}\left(\rho_{1}\right) / h_{1}\left(\rho_{1}\right)\right) \leq K, \rho_{1} \in\left[x_{1}, \zeta\right]$, then the following inequality holds:

$$
\left({ }_{x_{1}} \mathfrak{\mathfrak { I }}^{\tau} \mathcal{U}_{1}^{\delta}(\zeta)\right)^{(1 / \delta)}+\left({ }_{x_{1}} \mathfrak{\mathfrak { I }}^{\tau} \mathcal{U}_{2}^{\delta}(\zeta)\right)^{(1 / \delta)} \leq \frac{(1+K)(k+2)}{(k+1)(K+1)}\left({ }_{x_{1}} \mathfrak{\mathfrak { I }}^{\tau}\left(\mathcal{U}_{1}+\mathcal{U}_{2}\right)^{\delta}(\zeta)\right)^{(1 / \delta)}
$$

Proof. Under the given hypothesis of Theorem 5, $\left(\mathscr{U}_{1}\left(\rho_{1}\right) / \mathcal{U}_{2}\left(\rho_{1}\right)\right) \leq K, \rho_{1} \in\left[x_{1}, \zeta\right], \zeta>0$, we have

$$
(K+1)^{\delta} \mathscr{U}_{1}^{\delta}\left(\rho_{1}\right) \leq K^{\delta}\left(\mathscr{U}_{1}+\mathscr{U}_{2}\right)^{\delta}\left(\rho_{1}\right) .
$$

Consider a function:

$$
\Lambda\left(\zeta, \rho_{1}\right)=\frac{1}{\Gamma_{\mathscr{F}}^{\alpha}(\tau)}\left(S_{\mathscr{F}}^{\alpha}(\zeta)-S_{\mathscr{F}}^{\alpha}\left(\rho_{1}\right)\right)^{\tau-\alpha}
$$

We conclude that the function $\Lambda\left(\zeta, \rho_{1}\right)$ is positive for all $\rho_{1} \in\left(x_{1}, \zeta\right), 0 \leq x_{1}<\zeta \leq x_{2}$, as each term of $\Lambda\left(\zeta, \rho_{1}\right)$ defined in (15) is positive in view of hypothesis of Theorem 5.

Therefore, conducting product on both sides of (14) by $\Lambda\left(\zeta, \rho_{1}\right)$ and integrating the estimated inequality with respect to $\rho_{1}$ from $S_{\mathscr{F}}^{\alpha}(a)$ to $S_{\mathscr{F}}^{\alpha}(\zeta)$, we have

$$
\begin{aligned}
& \frac{(K+1)^{\delta}}{\Gamma_{\mathscr{F}}^{\alpha}(\tau)} \int_{x_{1}}^{\zeta}\left(S_{\mathscr{F}}^{\alpha}(\zeta)-S_{\mathscr{F}}^{\alpha}\left(\rho_{1}\right)\right)^{\tau-\alpha} \mathcal{U}_{1}^{\delta}\left(\rho_{1}\right) d_{\mathscr{F}}^{\alpha} \rho_{1} \\
& \quad \leq \frac{K^{\delta}}{\Gamma_{\mathscr{F}}^{\alpha}(\tau)} \int_{x_{1}}^{\zeta}\left(S_{\mathscr{F}}^{\alpha}(\zeta)-S_{\mathscr{F}}^{\alpha}\left(\rho_{1}\right)\right)^{\tau-\alpha}\left(\mathcal{U}_{1}+\mathscr{U}_{2}\right)^{\delta}\left(\rho_{1}\right) d_{\mathscr{F}}^{\alpha} \rho_{1},
\end{aligned}
$$

which can be written as

$$
x_{1} \mathfrak{T}^{\tau} \mathcal{U}_{1}^{\delta}(\zeta) \leq \frac{K^{\delta}}{(K+1)^{\delta}} x_{1} \mathfrak{T}^{\tau, \eta}\left(\mathcal{U}_{1}+\mathscr{U}_{2}\right)^{\delta}(\zeta)
$$

Hence, it follows that 


$$
\left({ }_{x_{1}} \mathfrak{\mathfrak { T }}^{\tau} \mathcal{U}_{1}^{\delta}(\zeta)\right)^{(1 / \delta)} \leq \frac{K}{(K+1)}\left({ }_{x_{1}} \mathfrak{\mathfrak { I }}^{\tau}\left(\mathcal{U}_{1}+\mathscr{U}_{2}\right)^{\delta}(\zeta)\right)^{(1 / \delta)}
$$

Now, utilizing the condition $k \mathscr{U}_{1}\left(\rho_{1}\right) \leq \mathscr{U}_{2}\left(\rho_{1}\right)$, we have

$$
\left(1+\frac{1}{k}\right) \mathscr{U}_{2}\left(\rho_{1}\right) \leq \frac{1}{k}\left(\mathscr{U}_{1}\left(\rho_{1}\right)+\mathscr{U}_{2}\left(\rho_{1}\right)\right) .
$$

It follows that

$$
\left(1+\frac{1}{k}\right)^{\delta} \mathscr{U}_{2}^{\delta}\left(\rho_{1}\right) \leq\left(\frac{1}{k}\right)^{\delta}\left(\mathscr{U}_{1}\left(\rho_{1}\right)+\mathscr{U}_{2}\left(\rho_{1}\right)\right)^{\delta} .
$$

Again, conducting product on both sides of (20) by $\Lambda\left(\zeta, \rho_{1}\right)$ and integrating the estimated inequality with respect to $\rho_{1}$ from $S_{\mathscr{F}}^{\alpha}\left(x_{1}\right)$ to $S_{\mathscr{F}}^{\alpha}(\zeta)$, we obtain

$$
\left({ }_{x_{1}} \mathfrak{T}^{\tau} \mathcal{U}_{2}^{\delta}(\zeta)\right)^{(1 / \delta)} \leq \frac{1}{(k+1)}\left({ }_{x_{1}} \mathfrak{T}^{\tau}\left(\mathcal{U}_{1}+\mathcal{U}_{2}\right)^{\delta}(\zeta)\right)^{(1 / \delta)}
$$

Thus, by adding inequalities (18) and (21) yields the desired inequality.

Theorem 6. Let $\tau \in \mathbb{C}, \mathfrak{R}(\tau)>0, \delta \geq 1$ and let $\mathcal{U}_{1}$ and $\mathcal{U}_{2} \in C_{\mathscr{F}}^{\alpha}\left[x_{1}, x_{2}\right]$ ( $\alpha$-order differentiable functions on $\left.\left[x_{1}, x_{2}\right]\right)$ be two positive functions on $[0, \infty)$ such that, for all $\zeta>0, \quad x_{1} \mathfrak{J}^{\tau} g_{1}^{\delta}(\zeta)<\infty \quad$ and ${ }_{x} \mathfrak{\Im}^{\tau} h^{\delta}(\zeta)<\infty$. If $0<k \leq\left(g_{1}\left(\rho_{1}\right) / h\left(\rho_{1}\right)\right) \leq K, \rho_{1} \in\left[x_{1}, \zeta\right]$, then the following inequality holds:

$$
\begin{aligned}
& \left({ }_{x_{1}} \mathfrak{T}^{\tau} \mathcal{U}_{1}^{\delta}(\zeta)\right)^{(2 / \delta)}+\left({ }_{x_{1}} \mathfrak{T}^{\tau} \mathcal{U}_{2}^{\delta}(\zeta)\right)^{(2 / \delta)} \\
& \quad \geq\left(\frac{(K+1)(k+1)}{K}-2\right)\left({ }_{x_{1}} \mathfrak{T}^{\tau} \mathcal{U}_{1}^{\delta}(\zeta)\right)^{(1 / \delta)}\left({ }_{x_{1}} \mathfrak{T}^{\tau} \mathcal{U}_{2}^{\delta}(\zeta)\right)^{(1 / \delta)}
\end{aligned}
$$

Proof. The multiplication of inequalities (18) and (21) yields

$$
\begin{aligned}
& \left(\frac{(K+1)(k+1)}{M}\right)\left({ }_{x_{1}} \mathfrak{T}^{\tau} \mathcal{U}_{1}^{\delta}(\zeta)\right)^{(1 / \delta)}\left({ }_{x_{1}} \mathfrak{\mathfrak { T }}^{\tau, \eta} \mathscr{U}_{2}^{\delta}(\zeta)\right)^{(1 / \delta)} \\
& \quad \leq\left[\left({ }_{x_{1}} \mathfrak{\mathfrak { T }}^{\tau}\left(\mathcal{U}_{1}(\zeta)+\mathscr{U}_{2}(\zeta)\right)^{\delta}\right)^{(1 / \delta)}\right]^{2}
\end{aligned}
$$

By utilizing the Minkowski inequality to the right-hand side of [17], we have

$$
\begin{aligned}
{\left[\left({ }_{x_{1}} \mathfrak{I}^{\tau}\left(g_{(\zeta)+\mathscr{U}_{2}(\zeta)}\right)^{\delta}\right)^{(1 / \delta)}\right]^{2} } & \leq\left[\left({ }_{x_{1}} \mathfrak{I}^{\tau} \mathcal{U}_{1}^{\delta}(\zeta)\right)^{(1 / \delta)}+\left({ }_{x_{1}} \mathfrak{\mathfrak { T }}^{\tau} \mathcal{U}_{2}^{\delta}(\zeta)\right)^{(1 / \delta)}\right]^{2} \\
& \leq\left({ }_{x_{1}} \mathfrak{\mathfrak { T }}^{\tau} \mathcal{U}_{1}^{\delta}(\zeta)\right)^{(2 / \delta)}+\left({ }_{x_{1}} \mathfrak{T}^{\tau} \mathcal{U}_{2}^{\delta}(\zeta)\right)^{(2 / \delta)}+2\left({ }_{x_{1}} \mathfrak{T}^{\tau} \mathcal{U}_{1}^{\delta}(\zeta)\right)^{(1 / \delta)}\left({ }_{x_{1}} \mathfrak{T}^{\tau} \mathcal{U}_{2}^{\delta}(\zeta)\right)^{(1 / \delta)}
\end{aligned}
$$

Thus, from inequalities (23) and (24), we get the desired inequality (22).

\section{Certain Related Nonlocal Fractal Integral Inequalities on Fractal Sets}

This section is devoted to deriving certain related nonlocal fractal integral inequalities on the fractal set.

Theorem 7. Let $\tau \in \mathbb{C}, \mathfrak{R}(\tau)>0, r>1,(1 / \delta)+(1 / \sigma)=1$ and let $\mathcal{U}_{1}$ and $\mathcal{U}_{2} \in C_{\mathscr{F}}^{\alpha}\left[x_{1}, x_{2}\right]$ ( $\alpha$-order differentiable functions on $\left[x_{1}, x_{2}\right]$ ) be two positive functions on $[0, \infty)$ such that $x_{1} \mathfrak{T}^{\tau}\left[\mathcal{U}_{1}(\zeta)\right]<\infty$ and $x_{1} \mathfrak{T}^{\tau}\left[\mathcal{U}_{2}(\zeta)\right]<\infty$. If $0<k \leq\left(\stackrel{g}{g}\left(\rho_{1}\right) / h\left(\rho_{1}\right)\right) \leq K<\infty, \rho_{1} \in\left[x_{1}, \zeta\right], \zeta>x_{1}$, we have

$$
\begin{aligned}
& \left({ }_{x_{1}} \mathfrak{T}^{\tau} \mathcal{U}_{1}(\zeta)\right)^{(1 / \delta)}\left({ }_{x_{1}} \mathfrak{T}^{\tau} \mathscr{U}_{2}(\zeta)\right)^{(1 / \sigma)} \\
& \quad \leq\left(\frac{K}{k}\right)^{(1 / r s)}\left({ }_{x_{1}} \mathfrak{T}^{\tau}\left[\mathcal{U}_{1}(\vartheta)\right]^{(1 / \delta)}\left[\mathcal{U}_{2}(\vartheta)\right]^{(1 / \sigma)}\right) .
\end{aligned}
$$

Proof. Since $\left(\mathcal{U}_{1}\left(\rho_{1}\right) / \mathscr{U}_{2}\left(\rho_{1}\right)\right) \leq K<\infty, \rho_{1} \in\left[x_{1}, \zeta\right], \zeta>a$, therefore, we have

$$
\left[\mathcal{U}_{2}\left(\rho_{1}\right)\right]^{(1 / \sigma)} \geq K^{(-1 / \sigma)}\left[\mathcal{U}_{1}\left(\rho_{1}\right)\right]^{(1 / \sigma)} .
$$

It follows that

$$
\begin{aligned}
{\left[\mathcal{U}_{1}\left(\rho_{1}\right)\right]^{(1 / \delta)}\left[\mathcal{U}_{2}\left(\rho_{1}\right)\right]^{(1 / \sigma)} } & \geq K^{(-1 / \sigma)}\left[\mathcal{U}_{1}\left(\rho_{1}\right)\right]^{(1 / \delta)}\left[\mathcal{U}_{1}\left(\rho_{1}\right)\right]^{(1 / \sigma)} \\
& \geq K^{(-1 / \sigma)}\left[\mathcal{U}_{1}\left(\rho_{1}\right)\right]^{(1 / \delta)+(1 / \sigma)} \\
& \geq K^{(-1 / \sigma)}\left[\mathcal{U}_{1}\left(\rho_{1}\right)\right] .
\end{aligned}
$$

Conducting multiplication on both sides of (27) by $\Lambda\left(\zeta, \rho_{1}\right)$ where $\Lambda\left(\zeta, \rho_{1}\right)$ is defined by (15) and integrating the estimated inequality with respect to $\rho_{1}$ from $S_{\mathscr{F}}^{\alpha}\left(x_{1}\right)$ to $S_{\mathscr{F}}^{\alpha}(\zeta)$, we have

$$
\begin{aligned}
& \frac{1}{\Gamma_{\mathscr{F}}^{\alpha}(\tau)} \int_{x_{1}}^{\zeta}\left(S_{\mathscr{F}}^{\alpha}(\zeta)-S_{\mathscr{F}}^{\alpha}\left(\rho_{1}\right)\right)^{\tau-\alpha}\left[\mathcal{U}_{1}\left(\rho_{1}\right)\right]^{(1 / \delta)}\left[\mathcal{U}_{2}\left(\rho_{1}\right)\right]^{(1 / \sigma)} d_{\mathscr{F}}^{\alpha} \rho_{1} \\
& \geq \frac{K^{(-1 / \sigma)}}{\Gamma_{\mathscr{F}}^{\alpha}(\tau)} \int_{x_{1}}^{\zeta}\left(S_{\mathscr{F}}^{\alpha}(\zeta)-S_{\mathscr{F}}^{\alpha}\left(\rho_{1}\right)\right)^{\tau-\alpha} g\left(\rho_{1}\right) d_{\mathscr{F}}^{\alpha} \rho_{1} .
\end{aligned}
$$

It follows that

$$
{ }_{x_{1}} \mathfrak{T}^{\tau}\left[\left(\mathcal{U}_{1}(\zeta)\right)^{(1 / \delta)}[h(\zeta)]^{(1 / \sigma)}\right] \geq K^{(-1 / \delta)}\left[{ }_{x_{1}} \mathfrak{T}^{\tau} \mathscr{U}_{1}(\zeta)\right] .
$$

Consequently, we have 


$$
{ }_{x_{1}} \mathfrak{T}^{\tau}\left[\left(\mathcal{U}_{1}(\zeta)\right)^{(1 / \delta)}[h(\zeta)]^{(1 / \sigma)}\right] \geq K^{(-1 / r s)}\left[{ }_{x_{1}} \mathfrak{T}^{\tau} \mathscr{U}_{1}(\zeta)\right]^{(1 / \delta)} .
$$

On the contrary, $k \mathscr{U}_{1}\left(\rho_{1}\right) \leq \mathscr{U}_{2}\left(\rho_{1}\right), \rho_{1} \in\left[x_{1}, \zeta\right], \zeta>x_{1} ;$ therefore, we have

$$
\left[\mathcal{U}_{1}\left(\rho_{1}\right)\right]^{(1 / \delta)} \geq k^{(1 / \delta)}\left[\mathscr{U}_{2}\left(\rho_{1}\right)\right]^{(1 / \delta)} .
$$

It follows that

$$
\begin{aligned}
{\left[\mathscr{U}_{1}\left(\rho_{1}\right)\right]^{(1 / \delta)}\left[h\left(\rho_{1}\right)\right]^{(1 / \sigma)} } & \geq k^{(1 / \delta)}\left[\mathcal{U}_{1}\left(\rho_{1}\right)\right]^{(1 / \delta)}\left[\mathcal{U}_{2}\left(\rho_{1}\right)\right]^{(1 / \sigma)} \\
& \geq k^{(1 / \delta)}\left[\mathcal{U}_{2}\left(\rho_{1}\right)\right]^{(1 / \delta)+(1 / \sigma)} \\
& \geq k^{(1 / \delta)}\left[\mathcal{U}_{2}\left(\rho_{1}\right)\right] .
\end{aligned}
$$

Again, conducting multiplication on both sides of (32) by $\Lambda\left(\zeta, \rho_{1}\right)$ where $\Lambda\left(\zeta, \rho_{1}\right)$ is defined by (15) and integrating the estimated inequality with respect to $\rho_{1}$ from $S_{\mathscr{F}}^{\alpha}\left(x_{1}\right)$ to $S_{\mathscr{F}}^{\alpha}(\zeta)$, we have

$$
\begin{aligned}
& \frac{1}{\Gamma_{\mathscr{F}}^{\alpha}(\tau)} \int_{x_{1}}^{\zeta}\left(S_{\mathscr{F}}^{\alpha}(\zeta)-S_{\mathscr{F}}^{\alpha}\left(\rho_{1}\right)\right)^{\tau-\alpha}\left[\mathcal{U}_{1}\left(\rho_{1}\right)\right]^{(1 / \delta)}\left[\mathscr{U}_{2}\left(\rho_{1}\right)\right]^{(1 / \sigma)} d_{\mathscr{F}}^{\alpha} \rho_{1} \\
& \quad \geq \frac{k^{(1 / \delta)}}{\Gamma_{\mathscr{F}}^{\alpha}(\tau)} \int_{x_{1}}^{\zeta}\left(S_{\mathscr{F}}^{\alpha}(\zeta)-S_{\mathscr{F}}^{\alpha}\left(\rho_{1}\right)\right)^{\tau-\alpha} \mathcal{U}_{2}\left(\rho_{1}\right) d_{\mathscr{F}}^{\alpha} \rho_{1} .
\end{aligned}
$$

Hence, we can write

$$
\left({ }_{x_{1}} \mathfrak{T}^{\tau}\left[\left[\mathcal{U}_{1}(\zeta)\right]^{(1 / \delta)}\left[\mathscr{U}_{2}(9)\right]^{(1 / \sigma)}\right]\right)^{(1 / \delta)} \geq k^{(1 / r)}\left[{ }_{x_{1}} \mathfrak{T}^{\tau} \mathcal{U}_{1}(\zeta)\right]^{(1 / \sigma)} .
$$

Multiplying (30) and (34), we get the desired inequality.

Theorem 8. Let $\tau \in \mathbb{C}, \mathfrak{R}(\tau)>0, \delta>1,(1 / \delta)+(1 / \sigma)=1$ and let $\mathscr{U}_{1}$ and $\mathscr{U}_{2} \in C_{\mathscr{F}}^{\alpha}\left[x_{1}, x_{2}\right]$ ( $\alpha$-order differentiable functions on $\left[x_{1}, x_{2}\right]$ ) be two positive functions on $[0, \infty)$ such that ${ }_{x_{1}} \mathfrak{T}^{\tau}\left[\mathscr{U}_{1}^{\delta}(\zeta)\right]<\infty$ and ${ }_{x_{1}} \mathfrak{I}^{\tau}\left[\mathscr{U}_{2}^{\sigma}(\zeta)\right]<\infty$. If $0<k \leq$ $\left(\mathcal{U}_{1}\left(\rho_{1}\right)^{\delta} / \mathcal{U}_{2}\left(\rho_{1}\right)^{\sigma}\right) \leq K<\infty, \rho_{1} \in[a, \vartheta], \zeta>x_{1}$, we have

$$
\begin{aligned}
& \left({ }_{x_{1}} \mathfrak{T}^{\tau} \mathscr{U}_{1}^{\delta}(\zeta)\right)^{(1 / \delta)}\left({ }_{x_{1}} \mathfrak{T}^{\tau} \mathcal{U}_{2}^{\sigma}(\zeta)\right)^{(1 / \sigma)} \\
& \quad \leq\left(\frac{K}{k}\right)^{(1 / r s)}\left({ }_{x_{1}} \mathfrak{T}^{\tau}\left[\mathscr{U}_{1}(\zeta)\right]^{1 / \delta}\left[\mathscr{U}_{2}(\zeta)\right]^{1 / \sigma}\right) .
\end{aligned}
$$

Proof. Replacing $\mathscr{U}_{1}(\zeta)$ and $\mathscr{U}_{2}(\zeta)$ by $\mathscr{U}_{1}^{\delta}(\zeta)$ and $h_{1}^{\sigma}(\zeta)$, $x_{1}<\zeta \leq x_{2}$ in Theorem 7 , and we get the desired inequality (35).

Theorem 9. Let $\tau \in \mathbb{C}, \mathfrak{R}(\tau)>0, \delta>1,(1 / \delta)+(1 / \sigma)=1$ and let $\mathscr{U}_{1}$ and $\mathscr{U}_{2} \in C_{\mathscr{F}}^{\alpha}\left[x_{1}, x_{2}\right]$ ( $\alpha$-order differentiable functions on $\left[x_{1}, x_{2}\right]$ ) be two positive functions on $[0, \infty)$ such that ${ }_{x_{1}} \mathfrak{T}^{\tau}\left[\mathcal{U}_{1}^{\delta}(\zeta)\right]<\infty$ and ${ }_{x_{1}} \mathfrak{T}^{\tau}\left[\mathcal{U}_{2}^{\sigma}(\zeta)\right]<\infty$. If $0<k \leq$ $\left(\mathscr{U}_{1}\left(\rho_{1}\right)^{\delta} / \mathscr{U}_{2}\left(\rho_{1}\right)^{\sigma}\right) \leq K<\infty$ where $k, K \in \mathbb{R}, \quad \rho_{1} \in$ $\left[x_{1}, \zeta\right], \zeta>x_{1}$, then the following inequality for left nonlocal fractal integral with fractal support holds:

$$
\begin{aligned}
{ }_{x_{1}} \mathfrak{T}^{\tau}\left[\mathcal{U}_{1}(\zeta) \mathcal{U}_{2}(\zeta)\right] \leq & \frac{2^{\delta-1} K^{\delta}}{\delta(K+1)^{\delta} x_{1}} \mathfrak{T}^{\tau}\left[\mathcal{U}_{1}^{\delta}+h^{\delta}\right](\zeta) \\
& +\frac{2^{\sigma-1}}{\sigma(k+1)^{\sigma}} x_{1} \mathfrak{T}^{\tau}\left[\mathcal{U}_{1}^{\sigma}+\mathcal{U}_{2}^{\sigma}\right](\vartheta) .
\end{aligned}
$$

Proof. By the given hypothesis $\left(\mathscr{U}_{1}\left(\rho_{1}\right) / \mathscr{U}_{2}\left(\rho_{1}\right)\right) \leq K$, we have

$$
(K+1)^{\delta} \mathcal{U}_{1}^{\delta}\left(\rho_{1}\right) \leq K^{\delta}\left[\mathcal{U}_{1}+\mathscr{U}_{2}\right]^{\delta}\left(\rho_{1}\right) .
$$

Conducting multiplication on both sides of inequality (37) by $\Lambda\left(\zeta, \rho_{1}\right)$ where $\Lambda\left(\zeta, \rho_{1}\right)$ id defined by (15) and integrating the estimated inequality with respect to $\rho_{1}$ over $\left(S_{\mathscr{F}}^{\alpha}\left(x_{1}\right), S_{\mathscr{F}}^{\alpha}(\zeta)\right)$, we obtain

$$
\begin{aligned}
& \frac{(K+1)^{\delta}}{\Gamma_{\mathscr{F}}^{\alpha}(\tau)} \int_{x_{1}}^{\zeta}\left(S_{\mathscr{F}}^{\alpha}(\zeta)-S_{\mathscr{F}}^{\alpha}\left(\rho_{1}\right)\right)^{\tau-\alpha} \mathscr{U}_{1}^{\delta}\left(\rho_{1}\right) d_{\mathscr{F}}^{\alpha} \rho_{1} \\
& \quad \leq \frac{K^{\delta}}{\Gamma_{\mathscr{F}}^{\alpha}(\tau)} \int_{x_{1}}^{\zeta}\left(S_{\mathscr{F}}^{\alpha}(\zeta)-S_{\mathscr{F}}^{\alpha}\left(\rho_{1}\right)\right)^{\tau-\alpha}\left[\mathscr{U}_{1}+\mathscr{U}_{2}\right]^{\delta}\left(\rho_{1}\right) d_{\mathscr{F}}^{\alpha} \rho_{1} .
\end{aligned}
$$

It follows that

$$
{ }_{x_{1}} \mathfrak{T}^{\tau} \mathscr{U}_{1}^{\delta}(\zeta) \leq \frac{K^{\delta}}{(K+1)^{\delta}} x_{1} \mathfrak{T}^{\tau}\left[\mathscr{U}_{1}+\mathscr{U}_{2}\right]^{\delta}(\zeta) .
$$

On the contrary, using $k \leq\left(\mathscr{U}_{1}\left(\rho_{1}\right) / \mathscr{U}_{2}\left(\rho_{1}\right)\right), a \leq \rho_{1} \leq \zeta$, we have

$$
(k+1)^{\sigma} \mathcal{U}_{2}^{\sigma}\left(\rho_{1}\right) \leq\left[\mathscr{U}_{1}+\mathcal{U}_{2}\right]^{\sigma}\left(\rho_{1}\right) .
$$

Again, conducting multiplication on both sides of inequality (40) by $\Lambda\left(\zeta, \rho_{1}\right)$ where $\Lambda\left(\zeta, \rho_{1}\right)$ is defined by (15) and integrating the estimated inequality with respect to $\rho_{1}$ over $\left(S_{\mathscr{F}}^{\alpha}\left(x_{1}\right), S_{\mathscr{F}}^{\alpha}(\zeta)\right)$, we obtain

$$
{ }_{x_{1}} \mathfrak{T}^{\tau} \mathcal{U}_{2}^{\sigma}(\zeta) \leq \frac{1}{(k+1)^{\sigma}} x_{1} \mathfrak{T}^{\tau}\left[\mathscr{U}_{1}+\mathscr{U}_{2}\right]^{\sigma}(\zeta) .
$$

Now, using Young's inequality, we have

$$
\mathcal{U}_{1}\left(\rho_{1}\right) \mathcal{U}_{2}\left(\rho_{1}\right) \leq \frac{\mathcal{U}_{1}^{\delta}\left(\rho_{1}\right)}{\delta}+\frac{\mathcal{U}_{1}^{\sigma}\left(\rho_{1}\right)}{\sigma} .
$$

Taking product on both sides of inequality (40) by $\Lambda\left(\zeta, \rho_{1}\right)$ where $\Lambda\left(\zeta, \rho_{1}\right)$ id defined by $(15)$ and integrating the resultant identity with respect to $\rho_{1}$ over $S_{\mathscr{F}}^{\alpha}\left(x_{1}\right)$ to $S_{\mathscr{F}}^{\alpha}(\zeta)$, we obtain

$$
{ }_{x_{1}} \mathfrak{T}^{\tau} \mathscr{U}_{1}(\zeta) \mathcal{U}_{2}(\zeta) \leq \frac{1}{\delta}\left({ }_{x_{1}} \mathfrak{T}^{\tau} \mathscr{U}_{1}^{\delta}(\zeta)\right)+\frac{1}{\sigma}\left({ }_{x_{1}} \mathfrak{T}^{\tau} \mathcal{U}_{1}^{\sigma}(\zeta)\right) .
$$

With the aid of (39) and (41), (43) can be written as 


$$
\begin{aligned}
& { }_{x_{1}} \mathfrak{T}^{\tau} \mathscr{U}_{1}(\zeta) \mathcal{U}_{2}(\zeta) \leq \frac{1}{\delta}\left({ }_{x_{1}} \mathfrak{T}^{\tau} \mathcal{U}_{1}^{\delta}(\zeta)\right)+\frac{1}{\sigma}\left({ }_{x_{1}} \mathfrak{T}^{\tau} \mathcal{U}_{1}^{\sigma}(\zeta)\right) \\
& \quad \leq \frac{K^{\delta}}{\delta(K+1)^{\delta}} x_{1} \mathfrak{T}^{\tau}\left[\mathscr{U}_{1}+\mathscr{U}_{2}\right]^{\delta}(\zeta)+\frac{1}{\sigma(k+1)^{\sigma}}{ }_{x_{1}} \mathfrak{T}^{\tau}\left[\mathscr{U}_{1}+\mathscr{U}_{2}\right]^{\sigma}(\zeta) .
\end{aligned}
$$

Now, using the inequality $\left(\rho_{1}+\omega\right)^{\delta} \leq 2^{\sigma-1}$ $\left(\rho_{1}^{\delta}+\omega^{\delta}\right), \delta>1, \rho_{1}, \omega>0$, one can obtain

$$
{ }_{x_{1}} \mathfrak{T}^{\tau}\left[\mathscr{U}_{1}+\mathscr{U}_{2}\right]^{\delta}(\zeta) \leq_{x_{1}} \mathfrak{T}^{\tau}\left[\mathscr{U}_{1}^{\delta}+\mathscr{U}_{2}^{\delta}\right](\zeta)
$$

$$
{ }_{x_{1}} \mathfrak{T}^{\tau}\left[\mathscr{U}_{1}+\mathcal{U}_{2}\right]^{\sigma}(\zeta) \leq_{x_{1}} \mathfrak{T}^{\tau}\left[\mathcal{U}_{1}^{\sigma}+\mathcal{U}_{2}^{\sigma}\right](\zeta) .
$$

Hence, the proof of (36) can be followed from (44)-(46).

Theorem 10. Let $\tau \in \mathbb{C}, \mathfrak{R}(\tau)>0, \delta \geq 1$ and let $\mathscr{U}_{1}$ and $\mathscr{U}_{2} \in C_{\mathscr{F}}^{\alpha}\left[x_{1}, x_{2}\right] \quad$ ( $\alpha$-order differentiable functions on $\left.\left[x_{1}, x_{2}\right]\right)$ be two positive functions on $[0, \infty)$ such that ${ }_{x} \mathfrak{I}^{\tau}\left[\mathscr{U}_{2}^{\delta}(\zeta)\right]<\infty,{ }_{x} \mathfrak{I}^{\tau}\left[\mathcal{U}_{2}^{\sigma}(\zeta)\right]<\infty$. If $0<l<k \leq\left(\mathscr{U}_{1}\left(\rho_{1}\right) /\right.$ $\left.\mathscr{U}_{2}\left(\rho_{1}\right)\right) \leq K<\infty$ where $l, k, K \in \mathbb{R}, \zeta>a$, then we have

and

$$
\begin{aligned}
\frac{K+1}{K-k}\left({ }_{x_{1}} \mathfrak{T}^{\tau}\left[\mathcal{U}_{1}(\zeta)-l \mathcal{U}_{2}(\zeta)\right]\right) & \leq\left({ }_{x_{1}} \mathfrak{T}^{\tau} \mathcal{U}_{1}^{\delta}(\zeta)\right)^{(1 / \delta)}+\left({ }_{x_{1}} \mathfrak{T}^{\tau} \mathcal{U}_{2}^{\delta}(\zeta)\right)^{(1 / \delta)} \\
& \leq \frac{k+1}{k-l}\left({ }_{x_{1}} \mathfrak{T}^{\tau}\left[\mathscr{U}_{1}(\zeta)-l \mathcal{U}_{2}(\zeta)\right]\right)^{(1 / \delta)}
\end{aligned}
$$

Proof. Under the given hypothesis $0<l<k \leq\left(\mathcal{U}_{1}\left(\rho_{1}\right)\right)$ $\left.\mathscr{U}_{2}\left(\rho_{1}\right)\right) \leq K<\infty$, we have

$k l \leq K l \Rightarrow k l+m \leq k l+K \leq K l+K \Rightarrow(K+1)(k-l) \leq(k+1)(k-l)$.

It can be written as

$$
\frac{(K+1)}{(K-l)} \leq \frac{(k+1)}{(k-l)} .
$$

Also, we have

$$
k-l \leq \frac{\mathscr{U}_{1}\left(\rho_{1}\right)-l \mathcal{U}_{2}\left(\rho_{1}\right)}{\mathcal{U}_{2}\left(\rho_{1}\right)} \leq K-l .
$$

It follows that

$$
\frac{\left(\mathscr{U}_{1}\left(\rho_{1}\right)-l \mathscr{U}_{2}\left(\rho_{1}\right)\right)^{\delta}}{(K-l)^{\delta}} \leq \mathscr{U}_{2}^{\delta}\left(\rho_{1}\right) \leq \frac{\left(\mathscr{U}_{1}\left(\rho_{1}\right)-l \mathcal{U}_{2}\left(\rho_{1}\right)\right)^{\delta}}{(k-l)^{\delta}} .
$$

Also, we have

$$
\frac{1}{K} \leq \frac{\mathscr{U}_{2}\left(\rho_{1}\right)}{\mathcal{U}_{1}\left(\rho_{1}\right)} \leq \frac{1}{K} \Rightarrow \frac{k-l}{k l} \leq \frac{\mathcal{U}_{1}\left(\rho_{1}\right)-l \mathcal{U}_{2}\left(\rho_{1}\right)}{l \mathcal{U}_{1}\left(\rho_{1}\right)} \leq \frac{k-l}{l K} .
$$

It follows that

$$
\begin{aligned}
\left(\frac{K}{K-l}\right)^{\delta} & \leq\left(\mathcal{U}_{1}\left(\rho_{1}\right)-l \mathcal{U}_{2}\left(\rho_{1}\right)\right)^{\delta} \leq \mathcal{U}_{1}^{\delta}\left(\rho_{1}\right) \leq\left(\frac{k}{k-l}\right)^{\delta} \\
& \leq\left(\mathcal{U}_{1}\left(\rho_{1}\right)-l \mathcal{U}_{2}\left(\rho_{1}\right)\right)^{\delta} .
\end{aligned}
$$

Conducting product on both sides of inequality (51) by $\Lambda\left(\zeta, \rho_{1}\right)$ where $\Lambda\left(\zeta, \rho_{1}\right)$ is defined by $(15)$ and integrating the resultant identity with respect to $\rho_{1}$ over $\left(S_{\mathscr{F}}^{\alpha}\left(x_{1}\right), S_{\mathscr{F}}^{\alpha}(\zeta)\right)$, we obtain

$$
\begin{aligned}
& \frac{1}{(K-l)^{\delta} \Gamma_{\mathscr{F}}^{\alpha}(\tau)} \int_{x_{1}}^{\zeta}\left(S_{\mathscr{F}}^{\alpha}(\zeta)-S_{\mathscr{F}}^{\alpha}\left(\rho_{1}\right)\right)^{\tau-\alpha}\left(\mathcal{U}_{1}\left(\rho_{1}\right)-l \mathcal{U}_{2}\left(\rho_{1}\right)\right)^{\delta} d_{\mathscr{F}}^{\alpha} \rho_{1} \leq \frac{1}{\Gamma_{\mathscr{F}}^{\alpha}(\tau)} \int_{x_{1}}^{\zeta}\left(S_{\mathscr{F}}^{\alpha}(\zeta)-S_{\mathscr{F}}^{\alpha}\left(\rho_{1}\right)\right)^{\tau-\alpha} \mathcal{U}_{2}^{\delta}\left(\rho_{1}\right) d_{\mathscr{F}}^{\alpha} \rho_{1} \\
& \quad \leq \frac{1}{(k-l)^{\delta} \Gamma_{\mathscr{F}}^{\alpha}(\tau)} \int_{x_{1}}^{\zeta}\left(S_{\mathscr{F}}^{\alpha}(\zeta)-S_{\mathscr{F}}^{\alpha}\left(\rho_{1}\right)\right)^{\tau-\alpha}\left(\mathcal{U}_{1}\left(\rho_{1}\right)-l \mathcal{U}_{2}\left(\rho_{1}\right)\right)^{\delta} d_{\mathscr{F}}^{\alpha} \rho_{1} .
\end{aligned}
$$

It follows that

$$
\frac{1}{(K-l)}\left({ }_{x_{1}} \mathfrak{T}^{\tau}\left(\mathcal{U}_{1}(\zeta) l \mathcal{U}_{2}(\zeta)\right)^{\delta}\right)^{(1 / \delta)} \leq\left({ }_{x_{1}} \mathfrak{T}^{\tau} \mathcal{U}_{2}^{\delta}(\zeta)\right)^{(1 / \delta)} \leq \frac{1}{(k-l)}\left(\left({ }_{x_{1}} \mathfrak{T}^{\tau}\left(\mathscr{U}_{1}(\zeta)-l \mathcal{U}_{2}(\zeta)\right)^{\delta}\right)^{(1 / \delta)}\right) .
$$


Again, conducting product on both sides of inequality (53) by $\Lambda\left(\zeta, \rho_{1}\right)$ where $\Lambda\left(\zeta, \rho_{1}\right)$ is defined by (15) and integrating the resultant identity with respect to $\rho_{1}$ over $\left(S_{\mathscr{F}}^{\alpha}\left(x_{1}\right), S_{\mathscr{F}}^{\alpha}(\zeta)\right)$, we obtain

$$
\begin{gathered}
\left(\frac{K}{K-l}\right)\left({ }_{x_{1}} \mathfrak{T}^{\tau}\left(\mathcal{U}_{1}(\zeta)-l \mathcal{U}_{2}(\zeta)\right)^{\delta}\right)^{(1 / \delta)} \leq\left({ }_{x_{1}} \mathfrak{T}^{\tau} \mathcal{U}_{1}^{\delta}(\zeta)\right)^{(1 / \delta)} \\
\leq\left(\frac{k}{k-l}\right)\left({ }_{x_{1}} \mathfrak{T}^{\tau}\left(\mathcal{U}_{1}(\zeta)-l \mathcal{U}_{2}(\zeta)\right)^{\delta}\right)^{(1 / \delta)} .
\end{gathered}
$$

Hence, by adding inequalities (55) and (56), we get the desired inequality (47).

Theorem 11. Let $\tau \in \mathbb{C}, \Re(\tau)>0, \delta \geq 1$ and let $\mathcal{U}_{1}$ and $\mathcal{U}_{2} \in C_{\mathscr{Y}}^{\alpha}\left[x_{1}, x_{2}\right]$ ( $\alpha$-order differentiable functions on $\left.\left[x_{1}, x_{2}\right]\right)$ be two positive functions on $[0, \infty)$ such that ${ }_{x_{1}} \mathfrak{T}^{\tau}\left[\mathcal{U}_{1}^{\delta}(\zeta)\right]<\infty,{ }_{1} \mathfrak{I}^{\tau}\left[\mathcal{U}_{2}^{\delta}(\zeta)\right]<\infty$. If $0 \leq \alpha \leq \mathcal{U}_{1}\left(\rho_{1}\right) \leq \mathscr{A}$ and $0 \leq \sigma \leq h_{1}\left(\rho_{1}\right) \leq \mathscr{B}$ for all $\rho_{1} \in\left[x_{1}, \zeta\right], \zeta>x_{1}$, then we have

$$
\begin{aligned}
& \left({ }_{x_{1}} \mathfrak{T}^{\tau} \mathscr{U}_{1}^{\delta}(\zeta)\right)^{(1 / \delta)}+\left({ }_{x_{1}} \mathfrak{T}^{\tau} \mathcal{U}_{2}^{\delta}(\zeta)\right)^{(1 / \delta)} \\
& \quad \leq \frac{\mathscr{A}(\alpha+\mathscr{B})+\mathscr{B}(\sigma+\mathscr{A})}{(\mathscr{A}+\sigma)(\mathscr{B}+\alpha)}\left({ }_{x_{1}} \mathfrak{T}^{\tau}\left[\mathscr{U}_{1}+\mathscr{U}_{2}\right]^{\delta}(\zeta)\right)^{(1 / \delta)} .
\end{aligned}
$$

Proof. Under the given hypothesis, we have

$$
\frac{1}{\mathscr{B}} \leq \frac{1}{U_{2}\left(\rho_{1}\right)} \leq \frac{1}{\sigma} .
$$
gives

The product of inequality (58) with $0 \leq \alpha \leq \mathscr{U}_{1}\left(\rho_{1}\right) \leq \mathscr{A}$

$$
\frac{\alpha}{\mathscr{B}} \leq \frac{\mathcal{U}_{1}\left(\rho_{1}\right)}{\mathcal{U}_{2}\left(\rho_{1}\right)} \leq \frac{\mathscr{A}}{\sigma} .
$$

From (59), we obtain

$$
\mathscr{U}_{2}^{\delta}\left(\rho_{1}\right) \leq\left(\frac{\mathscr{B}}{\alpha+\mathscr{B}}\right)^{\delta}\left(\mathscr{U}_{1}\left(\rho_{1}\right)+\mathscr{U}_{2}\left(\rho_{1}\right)\right)^{\delta}
$$

and

$$
\mathscr{U}_{1}^{\delta}\left(\rho_{1}\right) \leq\left(\frac{\mathscr{A}}{\sigma+\mathscr{A}}\right)^{\delta}\left(\mathscr{U}_{1}\left(\rho_{1}\right)+\mathscr{U}_{2}\left(\rho_{1}\right)\right)^{\delta} .
$$

Now, conducting product on both sides of (60) and (61), respectively, by $\Lambda\left(\zeta, \rho_{1}\right)$ where $\Lambda\left(\zeta, \rho_{1}\right)$ id is defined by (15) and integrating the estimated identity with respect to $\rho_{1}$ over $\left(S_{\mathscr{F}}^{\alpha}\left(x_{1}\right), S_{\mathscr{F}}^{\alpha}(\zeta)\right)$, we obtain

$$
\left({ }_{x_{1}} \mathfrak{T}^{\tau} \mathscr{U}_{2}^{\delta}(\zeta)\right)^{(1 / \delta)} \leq\left(\frac{\mathscr{B}}{\alpha+\mathscr{B}}\right)\left({ }_{x_{1}} \mathfrak{T}^{\tau}\left(\mathscr{U}_{1}(\zeta)+\mathscr{U}_{2}(\zeta)\right)^{\delta}\right)^{(1 / \delta)}
$$

and

$$
\left({ }_{x_{1}} \mathfrak{T}^{\tau} \mathcal{U}_{1}^{\delta}(\zeta)\right)^{(1 / \delta)} \leq\left(\frac{\mathscr{A}}{\sigma+\mathscr{A}}\right)\left({ }_{x_{1}} \mathfrak{T}^{\tau}\left(\mathcal{U}_{1}(\zeta)+\mathscr{U}_{2}(\zeta)\right)^{\delta}\right)^{(1 / \delta)}
$$

Hence, by adding (62) and (63), we get the desired proof.

Theorem 12. Let $\tau \in \mathbb{C}, \mathfrak{R}(\tau)>0, \delta \geq 1$ and let $\mathcal{U}_{1}$ and $\mathscr{U}_{2} \in C_{\mathscr{F}}^{\alpha}\left[x_{1}, x_{2}\right]$ ( $\alpha$-order differentiable functions on $\left.\left[x_{1}, x_{2}\right]\right)$ be two positive functions on $[0, \infty)$ such that $x_{1} \mathfrak{T}^{\mathfrak{T}}\left[\mathcal{U}_{1}(\zeta)\right]<\infty, x_{1} \mathfrak{T}^{\mathfrak{T}}\left[\mathscr{U}_{2}(\zeta)\right]<\infty$. If $0<k \leq\left(\mathcal{U}_{1}\left(\rho_{1}\right) /\right.$ $\left.h_{1}\left(\rho_{1}\right)\right) \leq K$ where $k, K \in \mathbb{R}$ for all $\rho_{1} \in\left[x_{1}, \zeta\right], \zeta>x_{1}$, then we have

$$
\begin{aligned}
\frac{1}{K}\left({ }_{x_{1}} \mathfrak{I}^{\tau} \mathscr{U}_{1}(\zeta) \mathcal{U}_{2}(\zeta)\right) & \leq \frac{1}{(k+1)(K+1)}\left({ }_{x_{1}} \mathfrak{I}^{\tau}\left(\mathcal{U}_{1}(\zeta)+\mathscr{U}_{2}(\zeta)\right)^{2}\right) \\
& \leq \frac{1}{k}\left({ }_{x_{1}} \mathfrak{T}^{\tau} \mathscr{U}_{1}(\zeta) \mathcal{U}_{2}(\zeta)\right) .
\end{aligned}
$$

Proof. Under the given hypothesis, $0<k \leq\left(\mathcal{U}_{1}\left(\rho_{1}\right)\right)$ $\left.\mathcal{U}_{2}\left(\rho_{1}\right)\right) \leq K$, we have

$$
\mathscr{U}_{2}\left(\rho_{1}\right)(k+1) \leq \mathscr{U}_{2}\left(\rho_{1}\right)+\mathscr{U}_{1}\left(\rho_{1}\right) \leq \mathscr{U}_{2}\left(\rho_{1}\right)(K+1) .
$$

Also, we have $(1 / K) \leq\left(\mathcal{U}_{2}\left(\rho_{1}\right) / \mathcal{U}_{1}\left(\rho_{1}\right)\right) \leq(1 / K)$, which gives

$$
\mathscr{U}_{1}\left(\rho_{1}\right)\left(\frac{K+1}{K}\right) \leq \mathscr{U}_{1}\left(\rho_{1}\right)+\mathscr{U}_{2}\left(\rho_{1}\right) \leq \mathscr{U}_{1}\left(\rho_{1}\right)\left(\frac{k+1}{k}\right) .
$$

The multiplication of (65) and (66) yields

$$
\frac{\mathscr{U}_{1}\left(\rho_{1}\right) \mathscr{U}_{2}\left(\rho_{1}\right)}{K} \leq \frac{\left(\mathscr{U}_{1}\left(\rho_{1}\right)+\mathscr{U}_{2}\left(\rho_{1}\right)\right)^{2}}{(k+1)(K+1)} \leq \frac{\mathscr{U}_{1}\left(\rho_{1}\right) \mathscr{U}_{2}\left(\rho_{1}\right)}{k} .
$$

Now, conducting multiplication on both sides of inequality (67) by $\Lambda\left(\zeta, \rho_{1}\right)$ where $\Lambda\left(\zeta, \rho_{1}\right)$ id is defined by (15) and integrating the resultant identity with respect to $\rho_{1}$ over $\left(S_{\mathscr{F}}^{\alpha}\left(x_{1}\right), S_{\mathscr{F}}^{\alpha}(\zeta)\right)$, we have 


$$
\begin{aligned}
& \frac{1}{K \Gamma_{\mathscr{F}}^{\alpha}(\tau)} \int_{x_{1}}^{\zeta}\left(S_{\mathscr{F}}^{\alpha}(\zeta)-S_{\mathscr{F}}^{\alpha}\left(\rho_{1}\right)\right)^{\tau-\alpha} \mathcal{U}_{1}\left(\rho_{1}\right) \mathcal{U}_{2}\left(\rho_{1}\right) d_{\mathscr{F}}^{\alpha} \rho_{1} \\
& \quad \leq \frac{1}{(k+1)(K+1) \Gamma_{\mathscr{F}}^{\alpha}(\tau)} \int_{x_{1}}^{\zeta}\left(S_{\mathscr{F}}^{\alpha}(\zeta)-S_{\mathscr{F}}^{\alpha}\left(\rho_{1}\right)\right)^{\tau-\alpha}\left(\mathscr{U}_{1}\left(\rho_{1}\right)+\mathscr{U}_{2}\left(\rho_{1}\right)\right)^{2} d_{\mathscr{F}}^{\alpha} \rho_{1} \\
& \quad \leq \frac{1}{k \Gamma_{\mathscr{F}}^{\alpha}(\tau)} \int_{x_{1}}^{\zeta}\left(S_{\mathscr{F}}^{\alpha}(\zeta)-S_{\mathscr{F}}^{\alpha}\left(\rho_{1}\right)\right)^{\tau-\alpha} \mathscr{U}_{1}\left(\rho_{1}\right) \mathcal{U}_{2}\left(\rho_{1}\right) d_{\mathscr{F}}^{\alpha} \rho_{1} .
\end{aligned}
$$

It follows that

$$
\begin{aligned}
\frac{1}{K}\left({ }_{x_{1}} \mathfrak{T}^{\tau} \mathscr{U}_{1}(\zeta) \mathcal{U}_{2}(\zeta)\right) & \leq \frac{1}{(k+1)(K+1)}\left({ }_{x_{1}} \mathfrak{T}^{\tau}\left(\mathscr{U}_{1}(\zeta)+\mathscr{U}_{2}(\zeta)\right)^{2}\right) \\
& \leq \frac{1}{k}\left({ }_{x_{1}} \mathfrak{T}^{\tau} \mathscr{U}_{1}(\zeta) \mathcal{U}_{2}(\zeta)\right),
\end{aligned}
$$

which completes the desired proof.

Theorem 13. Let $\tau \in \mathbb{C}, \mathfrak{R}(\tau)>0, \delta \geq 1$ and let $\mathscr{U}_{1}$ and $\mathscr{U}_{2} \in C_{\mathscr{F}}^{\alpha}\left[x_{1}, x_{2}\right]$ ( $\alpha$-order differentiable functions on $\left.\left[x_{1}, x_{2}\right]\right)$ be two positive functions on $[0, \infty)$ such that ${ }_{x_{1}} \mathfrak{T}^{\tau}\left[\mathscr{U}_{1}(\zeta)\right]<\infty$ and ${ }_{x_{1}} \mathfrak{T}^{\tau}\left[\mathscr{U}_{2}(\zeta)\right]<\infty$. If $0<k \leq\left(\mathscr{U}_{1}\left(\rho_{1}\right) /\right.$ $\left.h_{1}\left(\rho_{1}\right)\right) \leq K$ where $k, K \in \mathbb{R}$ for all $\rho_{1} \in\left[x_{1}, \zeta\right], \zeta>x_{1}$, then the following inequality for the left nonlocal fractal integral on fractal set holds:

$$
\begin{aligned}
& \left({ }_{x_{1}} \mathfrak{T}^{\tau} \mathcal{U}_{1}^{\delta}(\zeta)\right)^{(1 / \delta)}+\left({ }_{x_{1}} \mathfrak{T}^{\tau} \mathcal{U}_{2}^{\delta}(\zeta)\right)^{(1 / \delta)} \\
& \quad \leq 2\left({ }_{x_{1}} \mathfrak{T}^{\tau} h^{\delta}\left(\mathcal{U}_{1}(\zeta), \mathscr{U}_{2}(\zeta)\right)\right),
\end{aligned}
$$

where $h\left(\mathscr{U}_{1}(\zeta), \mathscr{U}_{2} \quad(\zeta)\right)=\max \left\{K\left[((K / k)+1) \mathcal{U}_{1}\left(\rho_{1}\right)-\right.\right.$ $\left.\left.K \mathscr{U}_{2}\left(\rho_{1}\right)\right],\left((k+K) \mathscr{U}_{2}\left(\rho_{1}\right)-\mathscr{U}_{1}\left(\rho_{1}\right) / k\right)\right\}$.

Proof. Under the given hypothesis $0<k \leq\left(\mathcal{U}_{1}\left(\rho_{1}\right) / \mathscr{U}_{2}\left(\rho_{1}\right)\right) \leq K$ where $\rho_{1} \in\left[x_{1}, \zeta\right], \zeta>x_{1}$, we have

$$
0<k \leq K+k-\frac{\mathscr{U}_{1}\left(\rho_{1}\right)}{\mathcal{U}_{2}\left(\rho_{1}\right)}
$$

and

$$
K+k-\frac{\mathscr{U}_{1}\left(\rho_{1}\right)}{\mathcal{U}_{2}\left(\rho_{1}\right)} \leq K .
$$

From (71) and (72), we have

$$
\mathscr{U}_{2}\left(\rho_{1}\right)<\frac{(K+k) \mathscr{U}_{2}\left(\rho_{1}\right)-\mathscr{U}_{1}\left(\rho_{1}\right)}{k} \leq h\left(\mathscr{U}_{1}\left(\rho_{1}\right), \mathscr{U}_{2}\left(\rho_{1}\right)\right) \text {, }
$$

where $h\left(\mathscr{U}_{1}\left(\rho_{1}\right), \mathscr{U}_{2}\left(\rho_{1}\right)\right)=\max \left\{K\left[((K / k)+1) \mathscr{U}_{1}\left(\rho_{1}\right)-\right.\right.$ $\left.\left.K \mathscr{U}_{2}\left(\rho_{1}\right)\right],\left((k+K) \mathscr{U}_{2}\left(\rho_{1}\right)-\mathscr{U}_{1}\left(\rho_{1}\right) / k\right)\right\}$. Also, from the given hypothesis $0<(1 / K) \leq\left(\mathscr{U}_{2}\left(\rho_{1}\right) / \mathscr{U}_{1}\left(\rho_{1}\right)\right) \leq(1 / K)$, we have

$$
\frac{1}{K} \leq \frac{1}{K}+\frac{1}{k}-\frac{\mathscr{U}_{2}\left(\rho_{1}\right)}{\mathcal{U}_{1}\left(\rho_{1}\right)}
$$

and

$$
\frac{1}{K}+\frac{1}{k}-\frac{\mathcal{U}_{2}\left(\rho_{1}\right)}{\mathcal{U}_{1}\left(\rho_{1}\right)} \leq \frac{1}{k}
$$

From (74) and (75), we obtain

$$
\frac{1}{K} \leq \frac{((1 / K)+(k)) \mathcal{U}_{1}\left(\rho_{1}\right)-\mathcal{U}_{2}\left(\rho_{1}\right)}{\mathcal{U}_{1}\left(\rho_{1}\right)} \leq \frac{1}{k} .
$$

It follows that

$$
\begin{aligned}
\mathscr{U}_{1}\left(\rho_{1}\right) & =K\left(\frac{1}{K}+\frac{1}{k}\right) \mathcal{U}_{1}\left(\rho_{1}\right)-K \mathscr{U}_{2}\left(\rho_{1}\right) \\
& =\frac{K(K+k) g\left(\rho_{1}\right)-K^{2} k \mathcal{U}_{2}\left(\rho_{1}\right)}{k K} \\
& =\left(\frac{K}{k}+1\right) \mathcal{U}_{1}\left(\rho_{1}\right)-K \mathcal{U}_{2}\left(\rho_{1}\right) \\
& =K\left[\left(\frac{K}{k}+1\right) \mathcal{U}_{1}\left(\rho_{1}\right)-K \mathcal{U}_{2}\left(\rho_{1}\right)\right] \\
& \leq h\left(\mathcal{U}_{1}\left(\rho_{1}\right), \mathscr{U}_{2}\left(\rho_{1}\right)\right) .
\end{aligned}
$$

From (73) and (77), we can write

$$
\mathcal{U}_{1}^{\delta}\left(\rho_{1}\right) \leq h^{\delta}\left(g\left(\rho_{1}\right), h\left(\rho_{1}\right)\right)
$$

and

$$
h^{\delta}\left(\rho_{1}\right) \leq h^{\delta}\left(g\left(\rho_{1}\right), h\left(\rho_{1}\right)\right) .
$$

Now, conducting multiplication on both sides of (78) and (79), respectively, by $\Lambda\left(\zeta, \rho_{1}\right)$ where $\Lambda\left(\zeta, \rho_{1}\right)$ id is defined by (15) and integrating the resultant identity with respect to $\rho_{1}$ over $\left(S_{\mathscr{F}}^{\alpha}\left(x_{1}\right), S_{\mathscr{F}}^{\alpha}(\zeta)\right)$, we obtain

$$
\begin{aligned}
& \frac{1}{\Gamma_{\mathscr{F}}^{\alpha}(\tau)} \int_{x_{1}}^{\zeta}\left(S_{\mathscr{F}}^{\alpha}(\zeta)-S_{\mathscr{F}}^{\alpha}\left(\rho_{1}\right)\right)^{\tau-\alpha} \mathscr{U}_{1}^{\delta}\left(\rho_{1}\right) d_{\mathscr{F}}^{\alpha} \rho_{1} \\
& \quad \leq \frac{1}{\Gamma_{\mathscr{F}}^{\alpha}(\tau)} \int_{x_{1}}^{\zeta}\left(S_{\mathscr{F}}^{\alpha}(\zeta)-S_{\mathscr{F}}^{\alpha}\left(\rho_{1}\right)\right)^{\tau-\alpha} h\left(\mathcal{U}_{1}\left(\rho_{1}\right), \mathscr{U}_{2}\left(\rho_{1}\right)\right) d_{\mathscr{F}}^{\alpha} \rho_{1} .
\end{aligned}
$$

It follows that

$$
\left({ }_{x_{1}} \mathfrak{T}^{\tau} \mathscr{U}_{1}^{\delta}(\zeta)\right)^{(1 / \delta)} \leq\left({ }_{x_{1}} \mathfrak{T}^{\tau} h\left(\mathscr{U}_{1}(\zeta), \mathscr{U}_{2}(\zeta)\right)\right)^{(1 / \delta)} \text {. }
$$

Similarly, from (79), we obtain 


$$
\left({ }_{x_{1}} \mathfrak{T}^{\tau} \mathscr{U}_{2}^{\delta}(\zeta)\right)^{(1 / \delta)} \leq\left({ }_{x_{1}} \mathfrak{T}^{\tau} h^{\delta}\left(\mathscr{U}_{1}(\zeta), \mathscr{U}_{2}(\zeta)\right)\right)^{(1 / \delta)}
$$

Hence, by summing (81) and (82), we obtain the required proof.

Remark 2. We note that all results lead to standard fractional calculus by setting $\alpha=1$, that is, $S_{\mathscr{F}}^{\alpha}(x)=x$.

\section{Concluding Remarks}

In this present investigation, we presented the nonlocal reverse Minkowski's inequalities and some other inequalities for nonlocal fractal integral operator on fractal sets. The special cases of this work can be found in the work of $[51,58,59]$.

\section{Data Availability}

No data were used in this study.

\section{Conflicts of Interest}

The authors declare that they have no conflicts of interest.

\section{References}

[1] V. V. Uchaikin, Fractional Derivatives for Physicists and Engineers Volumn 1 Background and Theory, Springer, Berlin, Germany, 2013.

[2] T. Sandev and Z. Tomovski, Fractional Equations and Models: Theory and Applications, Springer Nature, vol. 61, , 2019.

[3] D. Baleanu, K. Diethelm, E. Scalas, and J. Trujillo, Fractional Calculus: Models and Numerical Methods. Ser. on Complexity, Nonlinearity and Chaos, World Scientific, New York, NY, USA, 2012.

[4] S. G. Samko, A. A. Kilbas, and O. I. Marichev, Fractional Integrals and Derivatives Theory and Applications, Gordon and Breach, New York, NY, USA, 1993.

[5] I. Podlubny, Fractional Differential Equations, Academic Press, New York, NY, USA, 1999.

[6] A. A. Kilbas, H. M. Srivastava, and J. J. Trujillo, Theory and Applications of Fractional Differential Equations, NorthHolland Mathematics Studies, Vol. 207, Elsevier, Amsterdam, Netherlands, 2006.

[7] U. Skwara, J. Martins, P. Ghaffari et al., "Applications of fractional calculus to epidemiological models," AIP Conference Proceedings-American Institute of Physics, vol. 1479, pp. 1339-1342, 2012.

[8] B. J. West, M. Bologna, and P. Grigolini, Physics Fractal Operators, Springer, New York, NY, USA, 2003.

[9] S. Das, Functional Fractional Calculus, Springer Science Business Media, Berlin, Germany, 2011.

[10] D. Baleanu, A. K. Golmankhaneh, A. K. Golmankhaneh, and R. R. Nigmatullin, "Newtonian law with memory," Nonlinear Dynamics, vol. 60, no. 1-2, pp. 81-86, 2010.

[11] A. K. Golmankhaneh, Investigations in Dynamics: With Focus on Fractional Dynamics, LAP Lambert Academic Publishing, Saarbrucken, Germany, 2012.

[12] B. B. Mandelbrot, The Fractal Geometry of Nature, W. H. Freeman and Company, New York, NY, USA, 1977.

[13] K. Falconer, Techniques in Fractal Geometry, John Wiley and Sons, New York, NY, USA, 1997.
[14] J. Kigami, Analysis on Fractals, Cambridge University Press, New York, NY, USA, 2001.

[15] U. Freiberg and M. Zähle, "Harmonic calculus on fractals-a measure geometric approach I," Potential Analysis, vol. 16, no. 3, pp. 265-277, 2002.

[16] R. S. Strichartz, Differential Equations on Fractals: A Tutorial, Princeton University Press, Princeton, NJ, USA, 2006.

[17] K. Falconer, Techniques in Fractal Geometry, John Wiley and Sons, Hoboken, NJ, USA, 1997.

[18] M. T. Barlow and E. A. Perkins, "Brownian motion on the Sierpinski gasket," Probability Theory and Related Fields, vol. 79, no. 4, pp. 543-623, 1988.

[19] F. H. Stillinger, "Axiomatic basis for spaces with noninteger dimension," Journal of Mathematical Physics, vol. 18, no. 6, pp. 1224-1234, 1977.

[20] A. S. Balankin, "A continuum framework for mechanics of fractal materials I: from fractional space to continuum with fractal metric," The European Physical Journal B volume, vol. 88, p. 90, 2015.

[21] M. Zubair, M. J. Mughal, and Q. A. Naqvi, Electromagnetic Fields and Waves in Fractional Dimensional Space, Springer, New York, NY, USA, 2012.

[22] R. P. Alonso, T. Alexander, and C. J. Po-Chou, Analysis, Probability And Mathematical Physics on Fractals, vol. 5Singapore, World Scientific.

[23] P. Mattila, Geometry of Sets and Measures in Euclidean Spaces: Fractals and Rectifiability, Cambridge university press, Cambridge, England, 1999.

[24] M. L. Lapidus and M. Van Frankenhuysen, Dynamical, Spectral, and Arithmetic Zeta Functions: AMS Special Session on Dynamical, Spectral, and Arithmetic Zeta Functions, Vol. 290, American Mathematical Society, Washington, D.C., USA, 2001.

[25] M. L. Lapidus, G. Radunović, and D. Žubrinić, Fractal Zeta Functions and Fractal Drums: Higher-Dimensional Theory of Complex Dimensions, Springer International Publishing, New York, NY, USA, 2017.

[26] K. Falconer, Fractal Geometry: Mathematical Foundations and Applications, John Wiley \& Sons, Hoboken, NJ, USA, Third edition, 2013.

[27] L. Bougoffa, "On Minkowski and Hardy integral inequalities," Journal of Inequalities in Pure and Applied Mathematics, vol. 7, no. 2, 2006.

[28] J. M. Blackledge, A. K. Evans, and M. J. Turner, Fractal geometry. Mathematical Methods, Algorithms, Applications, Woodhead Publishing, Cambridge, UK, 2002.

[29] L. Nottale and J. Schneider, "Fractals and nonstandard analysis," Journal of Mathematical Physics, vol. 25, no. 5, pp. 1296-1300, 1984.

[30] M. Czachor, "Waves along fractal coastlines: from fractal arithmetic to wave equations," Acta Physica Polonica B, vol. 50, no. 4, pp. 813-831, 2017.

[31] G. Edgar, Measure, Topology, and Fractal Geometry, Springer Science \& Business Media, Berlin, Germany, 2008.

[32] V. E. Tarasov, Fractional Dynamics: Applications of Fractional Calculus to Dynamics of Particles, Fields and Media, SpringerVerlag Berlin Heidelberg, Berlin, Germany, 2010.

[33] G. Braden, Fractal Time: The Secret of 2012 and a New World Age, Hay House Inc., Carlsbad, CA, USA, 2010.

[34] P. R. Massopust, Fractal Functions, Fractal Surfaces, and Wavelets, Academic Press, Cambridge, MA, USA, 2016.

[35] M. Bohner and A. C. Peterson, Advances in Dynamic Equations on Time Scales, Birkhäuser Basel, Basel, Switzerland, 2003. 
[36] A. Parvate and A. D. Gangal, "Calculus on fractal subsets of real line - I: formulation,” Fractals, vol. 17, no. 1, pp. 53-81, 2003.

[37] A. Parvate and A. D. Gangal, "Calculus on fractal subsets of real line - II: conjugacy with ordinary calculus," Fractals, vol. 19, no. 3, pp. 271-290, 2011.

[38] A. Parvate, S. Satin, and A. D. Gangal, "Calculus on fractal curves in Rn," Fractals, vol. 19, no. 1, pp. 15-27, 2009.

[39] A. K. Golmankhaneh, A. Fernandez, A. K. Golmankhaneh, and D. Baleanu, "Diffusion on middle- $\xi$ Cantor sets," Entropy, vol. 20, no. 7, p. 504, 2018.

[40] A. K. Golmankhaneh and C. Tunç, "Sumudu transform in fractal calculus," Applied Mathematics and Computation, vol. 350, pp. 386-401, 2019.

[41] A. K. Golmankhaneh and A. Fernandez, "Random variables and stable distributions on fractal cantor sets," Fractal and Fractional, vol. 3, no. 2, p. 31, 2019.

[42] S. E. Satin, A. Parvate, and A. D. Gangal, "Fokker-Planck equation on fractal curves," Chaos, Solitons \& Fractals, vol. 52, pp. 30-35, 2013.

[43] S. Satin and A. D. Gangal, "Langevin equation on fractal curves," Fractals, vol. 24, no. 3, p. 1650028, 2014.

[44] A. K. Golmankhaneh and A. S. Balankin, "Sub-and superdiffusion on Cantor sets: beyond the paradox," Physics Letters A, vol. 382, no. 14, pp. 960-967, 2018.

[45] A. K. Golmankhaneh and D. Baleanu, "About schrödinger equation on fractals curves imbedding in $R^{3}$," International Journal of Theoretical Physics, vol. 54, no. 4, pp. 1275-1282, 2015.

[46] Z. Dahmani and L. Tabharit, "On weighted Gruss type inequalities via fractional integrals," Journal of Advanced Research in Pure Mathematics, vol. 2, no. 4, pp. 31-38, 2010.

[47] Z. Dahmani, "New inequalities in fractional integrals," International Journal of Nonlinear Science, vol. 9, no. 4, pp. 493-497, 2010.

[48] S. S. Dragomir, "A Generalization of Grüss's inequality in inner product spaces and applications," Journal of Mathematical Analysis and Applications, vol. 237, no. 1, pp. 74-82, 1999.

[49] S. S. Dragomir, "Some integral inequalities of Gruss type," Indian Journal of Pure and Applied Mathematics, vol. 31, no. 4, pp. 397-415, 2002.

[50] E. Set, M. Tomar, and M. Z. Sarikaya, "On generalized Grüss type inequalities for k-fractional integrals," Applied Mathematics and Computation, vol. 269, pp. 29-34, 2015.

[51] Z. Dahmani, "On Minkowski and Hermite-Hadamard integral inequalities via fractional integration," Annals of Functional Analysis, vol. 1, no. 1, pp. 51-58, 2010.

[52] J. V. C. Sousa and E. Capelas de Oliveira, "The Minkowski's inequality by means of a generalized fractional integral," AIMS Mathematics, vol. 3, no. 1, pp. 131-147, 2018.

[53] V. L. Chinchane and D. B. Pachpatte, "New fractional inequalities via Hadamard fractional integral," International Journal of Functional Analysis, Operator Theory and Applications, vol. 5, pp. 165-176, 2013.

[54] S. Taf and K. Brahim, "Some new results using Hadamard fractional integral," International Journal of Nonlinear Analysis and Applications, vol. 7, pp. 103-109, 2016.

[55] E. Set, M. Özdemir, and S. Dragomir, "On the HermiteHadamard inequality and other integral inequalities involving two functions," Journal of Inequalities and Applications, vol. 2010, no. 1, Article ID 148102, 2010.
[56] A. K. Golmankhaneh and D. Baleanu, "Non-local integrals and derivatives on fractal sets with applications," Open Physics, vol. 14, no. 1, pp. 542-548, 2016.

[57] A. K. Golmankhaneh and D. Baleanu, "New derivatives on the fractal subset of real-line," Entropy, vol. 18, no. 1, pp. 1-12, 2016.

[58] W. T. Sulaiman, "Reverses of Minkowski's, Hölders, and Hardys integral inequalities," International Journal of Mathematics and Mathematical Sciences, vol. 1, pp. 14-24, 2012.

[59] B. Sroysang, "More on reverses of Minkowski's integral inequality," Mathematica Aeterna, vol. 3, pp. 597-600, 2013. 